\title{
Glutathione loaded solid lipid nanoparticles: Preparation and in vitro evaluation as delivery systems of the antioxidant peptide to immunocompetent fish cells
}

\author{
Adriana Trapani ${ }^{\mathrm{a}}$, Giuseppe Tripodo ${ }^{\mathrm{b}}$, Delia Mandracchia ${ }^{\mathrm{a}}$, Nicola Cioffi ${ }^{\mathrm{c}}$, \\ Nicoletta Ditaranto ${ }^{\mathrm{c}}$, Rebeca Cerezuela ${ }^{\mathrm{d}}$ and Maria Angeles Esteban ${ }^{\mathrm{d}, *}$ \\ a Department of Pharmacy-Drug Sciences, University of Bari “Aldo Moro”, via Orabona, Bari, Italy \\ ${ }^{\mathrm{b}}$ Department of Drug Sciences, University of Pavia, viale Taramelli, Pavia, Italia \\ c Department of Chemistry, University of Bari “Aldo Moro”, via Orabona, Bari, Italy \\ ${ }^{\mathrm{d}}$ Department of Cell Biology \& Histology, Faculty of Biology, Regional Campus of International \\ Excellence "Campus Mare Nostrum”, University of Murcia, Murcia, Spain
}

\begin{abstract}
.
BACKGROUND: Solid lipid nanoparticles (SLN) have not been used for peptide supply to fish cells.

OBJECTIVE: To evaluate the potential of SLN to deliver the antioxidant glutathione (GSH) to the primary cultures of head-kidney (HK) leucocytes of Sparus aurata $L$.

METHODS: SLN were produced using the amphiphilic lipid Gelucire ${ }^{\circledR}$ 50/13 according to the melt-emulsification method. In vitro stability and colorimetric studies for the antioxidant activity were carried out prior to biological assays on HK leucocytes.

RESULTS: SLN sonicated in acidic medium were stable up to 3 months at $4{ }^{\circ} \mathrm{C}$. A strong in vitro antioxidant activity effect for SLN was shown. The incubation of gilthead seabream HK leucocytes with GSH, GSSG or the sonicated and non-sonicated GSH-SLN (HAc) $_{\text {for }} 4 \mathrm{~h}$ had very few effects on $\mathrm{HK}$ cell activities. The phagocytic ability of HK leucocytes previously incubated with GSH was significantly increased in respect to the control cells.

CONCLUSIONS: The technique of melt-emulsification provided particles with high association efficiency in GSH and even below $100 \mathrm{~nm}$ in size. However, no stimulant properties were observed after incubation of HK leucocytes with SLN and possible hypotheses explaining the observed findings are discussed.
\end{abstract}

Keywords: Glutathione, antioxidant activity, solid lipid nanoparticles, fish leucocytes, teleosts

\section{Introduction}

To improve fish nutrition, different diet protocols have been recommended and, in each of them, the presence of antioxidant substances is crucial to guarantee fundamental metabolic processes. The supply of peptide and peptidomimetic agents with the diet for fish may represent a useful tool to prevent the risk of pathologies and, overall, to improve their health [1]. Among the peptide and peptidomimetic compounds, glutathione (GSH) has gained much interest since it is involved in the defence from metal

\footnotetext{
${ }^{*}$ Corresponding author: M.A. Esteban, Department of Cell Biology and Histology, Faculty of Biology, University of Murcia, 30100 Murcia, Spain. Tel.: +34 868887665; Fax: +34 868883963; E-mail: aesteban@um.es.
} 
pollution so that it is considered a biomarker of oxidative stress and, moreover, may improve the health performances of the farmed species due to its antioxidant activity [2-5].

As in humans, also for fish the opportunity to supply GSH via the oral route, on one hand, is the "golden standard" due to the high compliance of such route of administration. On the other hand, also for fish GSH limited oral absorption could be mainly attributed to the chemical and enzymatic degradation of the peptide in the gastrointestinal tract. More specifically, the thiol group of the cysteine moiety in GSH can be subjected to enzymatic ( $\gamma$-glutamyl-transpeptidase) and non-enzymatic $\mathrm{pH}$-dependent oxidation, leading to the formation of inactive products as the glutathione disulfide (GSSG). To increase GSH amounts in fish, and, consequently, to reinforce fish cell defence mechanisms, carrier systems are to be designed to protect this antioxidant agent from the gastrointestinal environment and from enzymatic degradation.

From a technological point of view, however, the incorporation of hydrophilic compounds in colloidal drug delivery systems with high loading efficiency still remains a challenge, mainly due to the leaking of the drug to the outer aqueous phase during nanocarrier production. For instance, via the use of double emulsion or microemulsion systems, the encapsulation of water soluble agents is obtained, although such procedures often require the use of organic solvents, with consequent toxicological issues arising from solvent residues. Particularly, in the case of nanocarriers such as solid lipid nanoparticles (SLN), the encapsulation of hydrophilic substances was already explored, demonstrating that, by adapting the emulsion/melt dispersion, also a lipophilic matrix can successfully load hydrophilic agents [6-9].

The aim of the present work was developed SLN containing GSH and evaluated their potential for the supply of this peptide to immunocompetent fish cells. In our previous study we could show that SLN loaded with a fluorescent probe are efficiently internalized by primary cultures of head-kidney (HK) of Sparus aurata L. leucocytes and that the extent of this internalization is incubation time and SLN concentration dependent [10]. In general, it should be noted that, to the best of our knowledge, SLN were not previously applied for fish diseases and it explains our current interest to explore the performance of SLN as delivery systems of biological active substances to fish cells [11]. To prepare GSH-loaded SLN, the previously adopted melt-emulsification method was followed being it simple and versatile enough. Moreover, we used Gelucire ${ }^{\circledR} 50 / 13$ as lipid phase which is an amphiphilic lipid able to self-emulsify forming microemulsions and it is considered non-toxic and safe (GRAS, generally regarded as safe status) excipient. Such features of Gelucire ${ }^{\circledR}$ 50/13 may allow to efficiently encapsulate the small peptide GSH in SLN-based on this lipid $[12,13]$. Data of the in vitro characterization are herein presented, including the physicochemical properties of SLN as well and the in vitro evaluation of the antioxidant activity of these nanocarriers. Finally, biological assays were carried out on HK leucocytes to evaluate the potential of new SLN for the selective delivery of the antioxidant compound GSH to fish cells.

\section{Materials and methods}

\subsection{Materials}

Gelucire $^{\circledR}$ 50/13 was kindly donated by Gattefossè (Milan, Italy). L-Glutathione reduced (GSH), Tween 85, acetic acid, 2,2-diphenyl-1-picrylhydrazyl (DPPH), cetyltrimethylammonium bromide, 3,3',5,5' - tetramethylbenzidine hydrochloride (TMB) phorbol myristate acetate (PMA), fluorescein isothiocyanate (FITC) and luminol were purchased by Sigma Aldrich (Spain). RPMI-1640 culture medium and foetal calf serum (FCS) were provided by Gibco/Invitrogen (USA). Penicillin and streptomycin were purchased by Flow Laboratories Ltd. (UK). Double distilled water (Carlo Erba, Italy) was used throughout the study. All other chemicals were of reagent grade. 


\subsection{Preparation of GSH loaded SLN}

a) Formulation of SLN without sonication. Following the melt-emulsification method [10, 14, 15], Gelucire ${ }^{\circledR} 50 / 13(60 \mathrm{mg})$ was melted at $70^{\circ} \mathrm{C}$ in a glass vial. In a separate glass vial, an aqueous solution $(1.37 \mathrm{ml}$ of water or a diluted acetic solution $(0.01 \%, \mathrm{v} / \mathrm{v}, 1.37 \mathrm{ml}))$ of the surfactant (Tween $85,60 \mathrm{mg}$ ) was used as aqueous phase and it was heated at $70^{\circ} \mathrm{C}$. Once the Gelucire ${ }^{\circledR} 50 / 13$ melting occurred, in the aqueous phase $6 \mathrm{mg}$ of GSH were poured and, then, the resulting mixture was added to the melted phase at $70^{\circ} \mathrm{C}$, forming an emulsion by homogenization at $12,300 \mathrm{rpm}$ for 2 min with an UltraTurrax model T25 apparatus (Janke and Kunkel, Germany). Then, the nanosuspension was cooled at room temperature and the resulting SLN were collected by centrifugation $(16,000 \times \mathrm{g}, 45 \mathrm{~min}$, Eppendorf 5415D, Germany). Thus, besides the unloaded SLN, the formulations containing GSH were

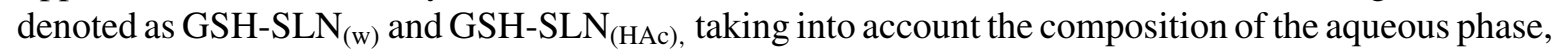
respectively. b) Formulation of SLN followed by sonication treatment. Only GSH-SLN arising from the use of diluted acetic acid and prepared as described in $a$ ) after Ultraturrax homogenization were subjected to the probe sonication (Branson 250 Sonifier, Branson) consisting of an output of energy of $20 \mathrm{~W}$ for $1 \mathrm{~min}$ at room temperature. Afterwards, the SLN were cooled at room temperature and collected by centrifugation $(16,000 \times \mathrm{g}, 45 \mathrm{~min}$, Eppendorf $5415 \mathrm{D}$, Germany).

\subsection{Physicochemical characterization of SLN}

The particle size and polydispersion index (PI) of all prepared SLN were determined in double distilled water by photon correlation spectroscopy (PCS) using a Zetasizer NanoZS (ZEN 3600, Malvern, UK). In the cuvette, each sample was diluted 1:1 (v/v) with double distilled water. The determination of the $\zeta$-potential was performed using laser Doppler anemometry (Zetasizer NanoZS, ZEN 3600, Malvern, UK) after dilution of 1:20 (v:v) in the presence of $\mathrm{KCl}(1 \mathrm{mM}, \mathrm{pH} 7)$ [16].

\subsection{Quantitative determination of GSH levels in SLN}

The amount of entrapped GSH was expressed as association efficiency (AE):

$\mathrm{AE}=$ Total weight of GSH-Weight of GSH in the supernatant/Total weight of GSH *100 where the total weight of GSH and the amount of GSH associated with the SLN supernatant were determined by HPLC analysis. In details, a reversed phase Synergy Hydro-RP $(25 \mathrm{~cm} \times 4.6 \mathrm{~mm} ; 4 \mu \mathrm{m}$ particles; Phenomenex, Torrance, CA) column in conjunction with a precolumn $\mathrm{C} 18$ insert was eluted with 1:99 (v:v) methanol:0.025 M phosphate buffer ( $\mathrm{pH} 2.7)$ in isocratic mode, with a flow rate of $0.7 \mathrm{ml} / \mathrm{min}$. Standard calibration curves were analyzed at $220 \mathrm{~nm}$ wavelength using $0.025 \mathrm{M}$ phosphate buffer ( $\mathrm{pH} 2.7)$ as solvent. Calibration curve linearity $\left(r^{2}>0.999\right)$ was maintained over the range of concentrations tested $(1000 \mu \mathrm{g} / \mathrm{ml}-10 \mu \mathrm{g} / \mathrm{ml})$. The retention times of GSH and its disulfide degradation product (Glutathione disulfide, GSSG) were 7.2 and $18 \mathrm{~min}$, respectively. Under these conditions, the LOQ limit was $2 \mu \mathrm{g} / \mathrm{ml}$ for both GSH and GSSG.

\subsection{Triton X assay}

To gain insight on the localization of the peptide GSH in the examined lipid carriers, the Triton X experiment was carried out. In details, the pellets arising from the centrifugation of GSH-SLN ${ }_{(H A c)}$ were treated with a Triton X solution $(2 \%, \mathrm{w} / \mathrm{v})$ to induce the breaking of the SLN matrix. The resulting mixture was vortexed and then analyzed by HPLC and, interestingly, the chromatogram showed the typical peak of reduced GSH, suggesting so the localization of the peptide inside the particles. 


\subsection{Stability studies of SLN}

GSH-SLN ${ }_{(\mathrm{HAc})}$, both sonicated and non-sonicated, were evaluated for their stability up to 3 months by monitoring particle size and zeta potential maintenance at $4{ }^{\circ} \mathrm{C}$ without any stirring [17]. The particle size and zeta values of the samples were determined at scheduled time intervals according to the protocol reported in Section 2.3. Each experiment was performed in triplicate.

\subsection{In vitro release studies}

Freshly prepared sonicated and not sonicated GSH-SLN $(\mathrm{HAc})$ were subjected to in vitro release studies up to $3 \mathrm{~h}$, by working in the presence of two phosphate buffered media without enzymes at $\mathrm{pH}$ 5 and $\mathrm{pH} 7$, respectively.

For each experiment, appropriate volumes of SLN, corresponding to an amount of GSH in the range $500-1000 \mu \mathrm{g} / \mathrm{ml}$, were put in Eppendorf tubes containing $1.8 \mathrm{ml}$ of buffer. The samples were incubated under mechanical agitation $(100 \mathrm{rpm})$ at $22^{\circ} \mathrm{C}[10]$ and at prefixed time intervals $(0,0.5,1,1.5,2,2.5$ and $3 \mathrm{~h}$ ) each sample was centrifuged $(16,000 \times \mathrm{g}$ for $45 \mathrm{~min})$. The supernatant was analyzed for the content of GSH using HPLC method described in Section 2.4. Each experiment was performed in triplicate.

\subsection{In vitro antioxidant activity of $G S H-S L N$}

The in vitro antioxidant activity of selected GSH-SLN, unloaded SLN and pure GSH was evaluated according to the 2,2-diphenyl-1-picrylhydrazyl (DPPH) radical test [18-20]. The DPPH • was dissolved in ethanol at the concentration of $0.001 \%(\mathrm{w} / \mathrm{v})$ to obtain a stock solution and, then, diluted up to reach the concentration equal to $8 \times 10^{-4} \%(\mathrm{w} / \mathrm{v}) .0 .5 \mathrm{ml}$ of each sample reacted with $2.5 \mathrm{ml}$ of the diluted solution of DPPH. for $60 \mathrm{~min}$ at room temperature under light protection. The absorbance changes were measured at the wavelength of $514 \mathrm{~nm}$ (Perkin-Elmer Lambda Bio20-UV-VIS spectrophotometer).

Blanks were constituted by filling the cuvettes with $3 \mathrm{ml}$ of DPPH. at the concentration of $8 \times 10^{-4} \%$ $(\mathrm{w} / \mathrm{v})$. Antioxidant activity was calculated from the Equation (1) and expressed in percentages:

$$
\mathrm{AA}=1-\left[\left(\mathrm{A}_{\mathrm{S}}\right) /\left(\mathrm{A}_{\mathrm{b}}\right)\right]
$$

Equation (1)

where AA is the antioxidant activity, $A_{s}$ represents the absorbance of the sample, and $A_{b}$ represents the absorbance of the blank cuvette.

\subsection{Animals}

Six specimens ( $20 \mathrm{~g}$ main weight and $6 \mathrm{~cm}$ main length) of the hermaphroditic protandrous seawater teleost gilthead seabream (S. aurata L.), obtained from a local farm (Murcia, Spain), were kept in re-circulating seawater aquaria $(250 \mathrm{~L})$ in the Marine Fish Facility at the University of Murcia. The water temperature was maintained at $20 \pm 2{ }^{\circ} \mathrm{C}$ with a flow rate of $900 \mathrm{~L} / \mathrm{h}$ and $28 \%$ salinity. The photoperiod was of $12 \mathrm{~h}$ light: $12 \mathrm{~h}$ dark and fish were fed with a commercial pellet diet (Skretting, Spain) at a rate of $2 \%$ body weight per day. Fish were allowed to acclimatise for 15 days before the start of the experimental trial. All experimental protocols were approved by the Ethical Committee of the University of Murcia. 


\subsection{Leucocyte isolation}

Specimens were weighted and bled (blood was collected from the caudal vein with an insulin syringe) and the HK (the main haemopoietic organ in this fish species, equivalent to mammalian bone marrow) was dissected. HK samples were cut into small fragments and transferred to $8 \mathrm{ml}$ of sRPMI [RPMI1640 culture medium supplemented with $0.35 \%$ sodium chloride (to adjust the medium's osmolarity to seabream plasma osmolarity of $353.33 \mathrm{mOs}$ ), $2 \% \mathrm{FCS}, 100 \mathrm{I} . \mathrm{U} . \mathrm{ml}^{-1}$ penicillin and $100 \mu \mathrm{g} / \mathrm{ml}$ streptomycin] for leucocyte isolation [21]. Cell suspensions were obtained by forcing fragments of the organ through a nylon mesh (mesh size $100 \mu \mathrm{m})$, washed twice $(400 \mathrm{~g} 10 \mathrm{~min})$, counted (Z2 Coulter Particle Counter) and adjusted to $2 \times 10^{7}$ cells $/ \mathrm{ml}$ in sRPMI. Cell viability was higher than $98 \%$, as determined by the Trypan blue exclusion test. All the cellular immune functions were performed only in viable cells.

\subsection{Incubation of leucocytes}

To study the effects of the particles on seabream HK leucocytes, cells (final concentration of $10^{7}$ cells $/ \mathrm{ml}$ ) were incubated without (control) or with $10 \mu \mathrm{g} / \mathrm{ml}$ of culture medium containing GSH, GSSG, GSH-SLN ${ }_{(\mathrm{HAc})}$, or sonicated GSH-SLN ${ }_{(\mathrm{HAc})}$. Incubation was performed at $25^{\circ} \mathrm{C}$ with $85 \%$ relative humidity and $5 \% \mathrm{CO}_{2}$ atmosphere for $4 \mathrm{~h}$.

\subsection{Leucocyte immune parameters}

\subsubsection{Peroxidase activity}

The peroxidase activity in HK leucocytes previously incubated with GSH, GSSG or SLN was measured according to [22]. $10^{6} \mathrm{HK}$ leucocytes in SRPMI were lysed with $0.002 \%$ cetyltrimethylammonium bromide and, after centrifugation ( $400 \mathrm{~g}, 10 \mathrm{~min}), 150 \mu \mathrm{l}$ of the supernatants were transferred to a fresh 96-well plate containing $25 \mu \mathrm{l}$ of $10 \mathrm{mM} \mathrm{TMB}$ and $5 \mathrm{mM} \mathrm{H}_{2} \mathrm{O}_{2}$. The colour-change reaction was stopped after $2 \mathrm{~min}$ by adding $50 \mu \mathrm{l}$ of $2 \mathrm{M}$ sulphuric acid and the optical density was read at $450 \mathrm{~nm}$ in a plate reader (BMG Labtech). Standard samples without serum or leucocytes, respectively, were used as blanks.

\subsubsection{Respiratory burst activity}

The respiratory burst activity of gilthead seabream HK leucocytes was studied by a chemiluminescence method [23]. Briefly, samples of $10^{6}$ leucocytes in sRPMI were placed in the wells of a flat-bottomed 96-well microtiter plate, to which $100 \mu \mathrm{l}$ of $\mathrm{HBSS}$ containing $1 \mu \mathrm{g} \mathrm{ml}^{-1}$ PMA and $10^{-4}$ $\mathrm{M}$ luminol were added. The plate was shaken and luminescence immediately detected in a plate reader for $1 \mathrm{~h}$ at $2 \mathrm{~min}$ intervals. The kinetics of the reactions were analysed and the maximum slope of each curve was calculated. Luminescence backgrounds were calculated using reagent solutions containing luminol but not PMA.

\subsubsection{Phagocytic activity}

The phagocytosis of Saccharomyces cerevisiae (strain S288C) by gilthead seabream HK leucocytes was studied by flow cytometry [24]. Heat-killed and lyophilized yeast cells were labelled with FITC washed and adjusted to $5 \times 10^{7}$ cells $\mathrm{ml}^{-1}$ of sRPMI. Phagocytosis samples consisted of $125 \mu \mathrm{l}$ of labelled-yeast cells and $100 \mu \mathrm{l}$ of HK leucocytes in sRPMI (6.25 yeast cells:1 leucocyte). Samples were mixed, centrifuged $\left(400 \mathrm{~g}, 5 \mathrm{~min}, 22^{\circ} \mathrm{C}\right)$, resuspended and incubated $\left(22^{\circ} \mathrm{C}, 30 \mathrm{~min}\right)$. At the end 
of the incubation time, samples were placed on ice to stop phagocytosis and $400 \mu \mathrm{l}$ ice-cold PBS was added to each sample. The fluorescence of the extracellular yeasts was quenched by adding $40 \mu \mathrm{l}$ ice-cold trypan blue ( $0.4 \%$ in PBS). Standard samples of FITC-labelled $S$. cerevisiae or HK leucocytes were included in each phagocytosis assay.

All samples were analysed in a flow cytometer (Becton Dickinson) with an argon-ion laser adjusted to $488 \mathrm{~nm}$. Analyses were performed on 3,000 cells, which were acquired at a rate of $300 \mathrm{cells} / \mathrm{s}$. Data were collected in the form of two-parameter side scatter (granularity) (SSC) and forward scatter (size) (FSC) and green fluorescence (FL1) dot plots or histograms were made on a computerised system. The fluorescence histograms represented the relative fluorescence on a logarithmic scale. The cytometer was set to analyse the phagocytic cells, showing highest SSC and FSC values. Phagocytic ability was defined as the percentage of cells with one or more ingested bacteria (green-FITC fluorescent cells) within the phagocytic cell population whilst the phagocytic capacity was the mean fluorescence intensity. The quantitative study of the flow cytometric results was made using the statistical option of the Lysis Software Package (Becton Dickinson).

\subsection{Statistical analysis}

In vitro data from different experimental groups were compared by a one-way ANOVA and differences were considered significant at $95 \%$ level of confidence $(p<0.05)$ using GraphPad Prism v. 5.00 computer program (GraphPad Software, Inc. CA, USA). Bonferroni tests were used for post-hoc contrast.

For biological evaluation, all measurements were performed on three replicates and the results in figures are expressed as mean \pm standard error (SEM) $(n=6)$. Data were statistically analysed by the t- Student test using SPSS 19 to determine differences between control and previously incubated groups. Differences were considered statistically significant when $p \leq 0.05$.

\section{Results}

\subsection{Physicochemical characterization of SLN}

Table 1 shows the mean particle size, polydispersion index (PI), zeta potential and association efficiency (AE) for unloaded- and GSH loaded-SLN. The particle size of the prepared SLN was in the range of 69-223 nm, where the smallest particles were obtained after sonication treatment. Moreover, the examined SLN were characterized by PI values around 0.4 suggesting a broad distribution of the same particles [25]. All zeta potential values were found negative, but in the case of SLN formulated starting from acetic phase the external charge of the SLN was slightly negative for both GSH-SLN ${ }_{(\mathrm{HAc})}$, and sonicated $\mathrm{GSH}-\mathrm{SLN}_{(\mathrm{HAc}),}(-4.0 \mathrm{mV}$ and $-2.5 \mathrm{mV}$, respectively). These zeta potential values were significantly different from the negative enough surface charge of the control particles $(p \leq 0.001)$. The association efficiencies resulted in the range 40.1-82.7\% with the maximum value of the peptide entrapped observed for GSH-SLN $(\mathrm{HAc})$.

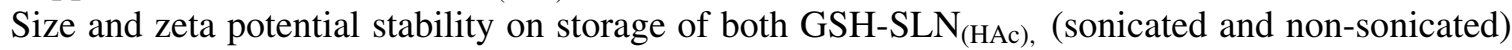
was also monitored up to 3 months at $4^{\circ} \mathrm{C}$. In terms of both size and zeta values, the most stable formulation was the sonicated GSH-SLN $(\mathrm{HAc})$ (Fig. 1). On the other hand, GSH-SLN $\mathrm{HAc}_{(\mathrm{H})}$ were seen to increase particle size and zeta values especially at the latest time points. Indeed, none of the tested suspensions was characterized by changes in their consistency, giving rise to aggregates or flocculation throughout the study. 
Table 1

Physicochemical properties of GSH containing SLN. Mean \pm S.D. are reported, $n=6$. Unloaded SLN in water and in diluted acetic acid were used as control for GSH-SLN $\left(\right.$ w) and for GSH-SLN $(\mathrm{HAc})$, respectively. $\left(^{*}\right) p \leq 0.05 ;\left(^{* *}\right) p \leq 0.001$

\begin{tabular}{|c|c|c|c|c|c|}
\hline Formulation & $\begin{array}{l}\text { Amount of } \\
\text { GSH (mg) }\end{array}$ & Size $(\mathrm{nm})$ & PI & $\begin{array}{c}\text { Zeta } \\
\text { Potential }(\mathrm{mV})\end{array}$ & A.E. $(\%)$ \\
\hline Control SLN $_{(w)}$ & - & $141( \pm 11)$ & $0.28-0.40$ & $-9.7( \pm 0.8)$ & - \\
\hline GSH-SLN $_{(w)}$ & 6.0 & $152( \pm 25)$ & $0.41-0.48$ & $-1.2( \pm 0.2)^{*}$ & $40.1( \pm 0.8)$ \\
\hline Control SLN (HAc) & - & $170( \pm 31)$ & $0.43-0.47$ & $-4.9( \pm 1.7)$ & - \\
\hline GSH-SLN $_{(\mathrm{HAc})}$ & 6.0 & $223( \pm 44)^{*}$ & $0.36-0.46$ & $-4.0( \pm 0.4)^{* *}$ & $82.7( \pm 2.9)$ \\
\hline Sonicated GSH-SLN ${ }_{(\mathrm{HAc})}$ & 6.0 & $69( \pm 10)^{* *}$ & $0.42-0.49$ & $-2.5( \pm 0.6)^{* *}$ & $53.6( \pm 9.6)$ \\
\hline
\end{tabular}
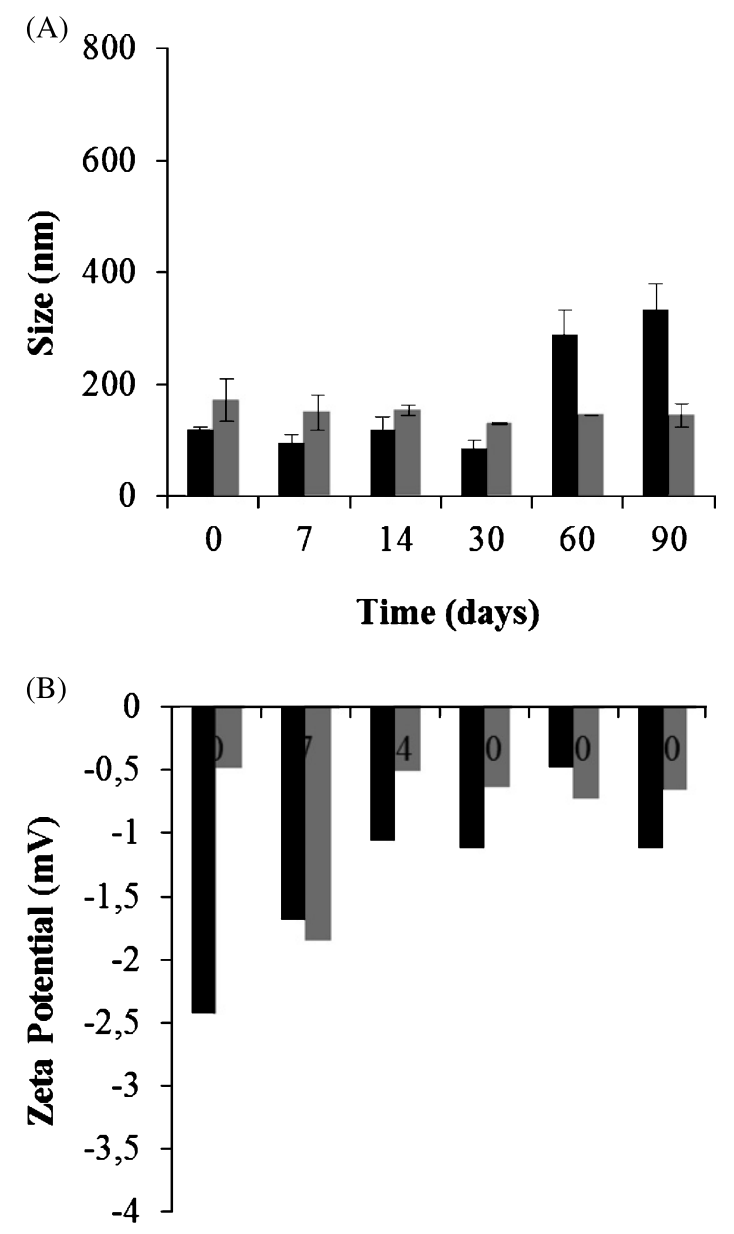

Time (days)

Fig. 1. Particle size (A) and zeta potential values (B) of GSH-SLN $\mathrm{HAc}_{(\mathrm{A})}$, both sonicated and non sonicated, upon incubation at $4^{\circ}$ C. Black bars: GSH-SLN ${ }_{(\mathrm{HAc})}$; grey bars: Sonicated GSH-SLN $\mathrm{HAc}_{(\mathrm{HAc})}$. 

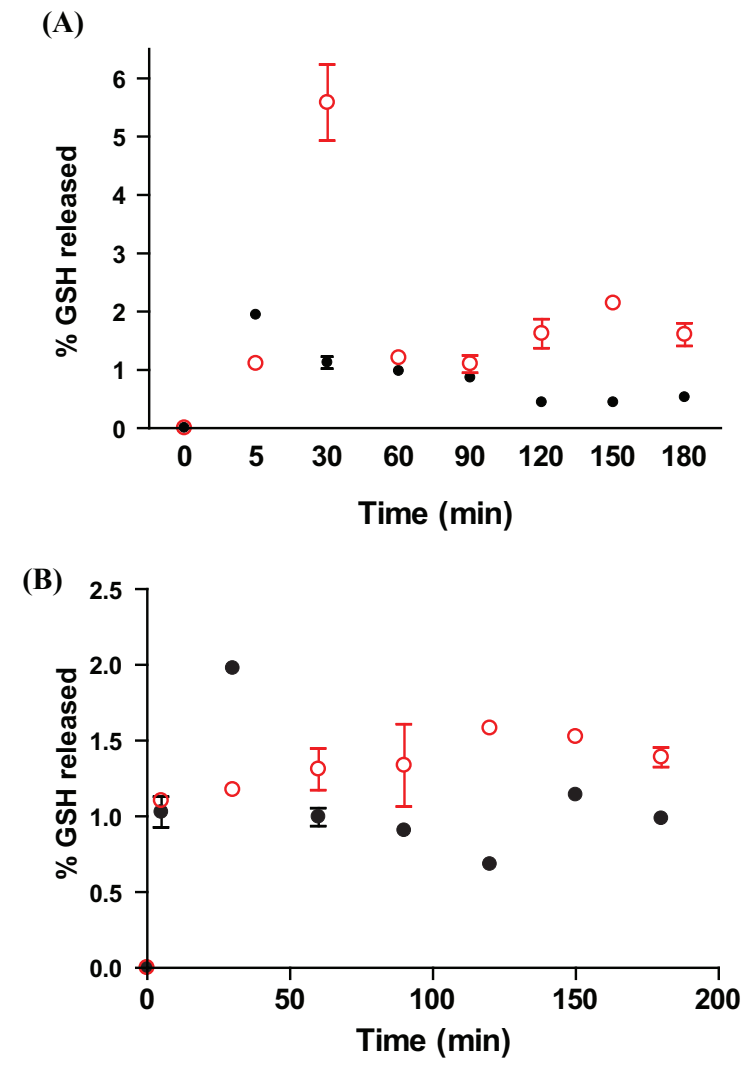

Fig. 2. In vitro release profile of GSH at (A) $\mathrm{pH} 5$ and (B) $\mathrm{pH} 7$ from GSH-SLN $\mathrm{HAc}_{(\mathrm{Ac})}$ (filled circles) and sonicated GSH$\operatorname{SLN}_{(\mathrm{HAc})}$ (unfilled circles).

\subsection{In vitro release studies of $S L N$}

At $22^{\circ} \mathrm{C}$ in both media represented by buffer without enzymes at $\mathrm{pH} 5$ and $\mathrm{pH}$ 7, GSH was released in little amount (around 1\%) from both sonicated and non-sonicated GSH-SLN (HAc) $_{\text {over }} 3 \mathrm{~h}$ of observation (Figs. 2A and B). Between the two formulations compared, sonicated GSH-SLN (HAc) $_{\text {provided }}$ a slightly higher percentage of the antioxidant peptide delivered in both the two buffered media.

\subsection{In vitro antioxidant activity of $G S H-S L N$}

To evaluate the in vitro ability of such formulations to keep intact the antioxidant activity of GSH, the spectrophotometric DPPH assay was carried out [18-20]. DPPH radical has a deep violet colour in solution, and gradually it became pale yellow in the presence of non-encapsulated GSH. As reported in Table 2, GSH aqueous solution taken as control provided $100 \%$ activity against DPPH radical as already observed by us [18]. In addition, both SLN containing GSH demonstrated 100\% antioxidant activity.

\subsection{Leucocyte immune parameters}

The incubation of HK leucocytes with GSH, GSSG or the SLN tested in the present work (sonicated and non-sonicated GSH-SLN $(\mathrm{HAc})$ ) for $4 \mathrm{~h}$ did not provoke any significant alteration neither in the 
Table 2

Antioxidant activity (\% AA) of free GSH dissolved in in Tween $85 / \mathrm{CH}_{3} \mathrm{COOH}(\mathrm{GSH})$, Control $\mathrm{SLN}_{(\mathrm{HAc})}$, GSH-SLN $(\mathrm{HAc})$ and sonicated GSH-SLN $\mathrm{GHAc}_{(\mathrm{H})}$. Data represent means $\pm \mathrm{S} . \mathrm{D}(n=3)$

\begin{tabular}{lc}
\hline Sample & $\%$ AA $( \pm S . D)$ \\
\hline GSH & $\geq 100$ \\
Control SLN $_{(H A c)}$ & $52.4( \pm 2.1)$ \\
GSH-SLN $_{(\mathrm{HAc})}$ & $\geq 100$ \\
Sonicated GSH-SLN $_{(\mathrm{HAc})}$ & $\geq 100$ \\
\hline
\end{tabular}
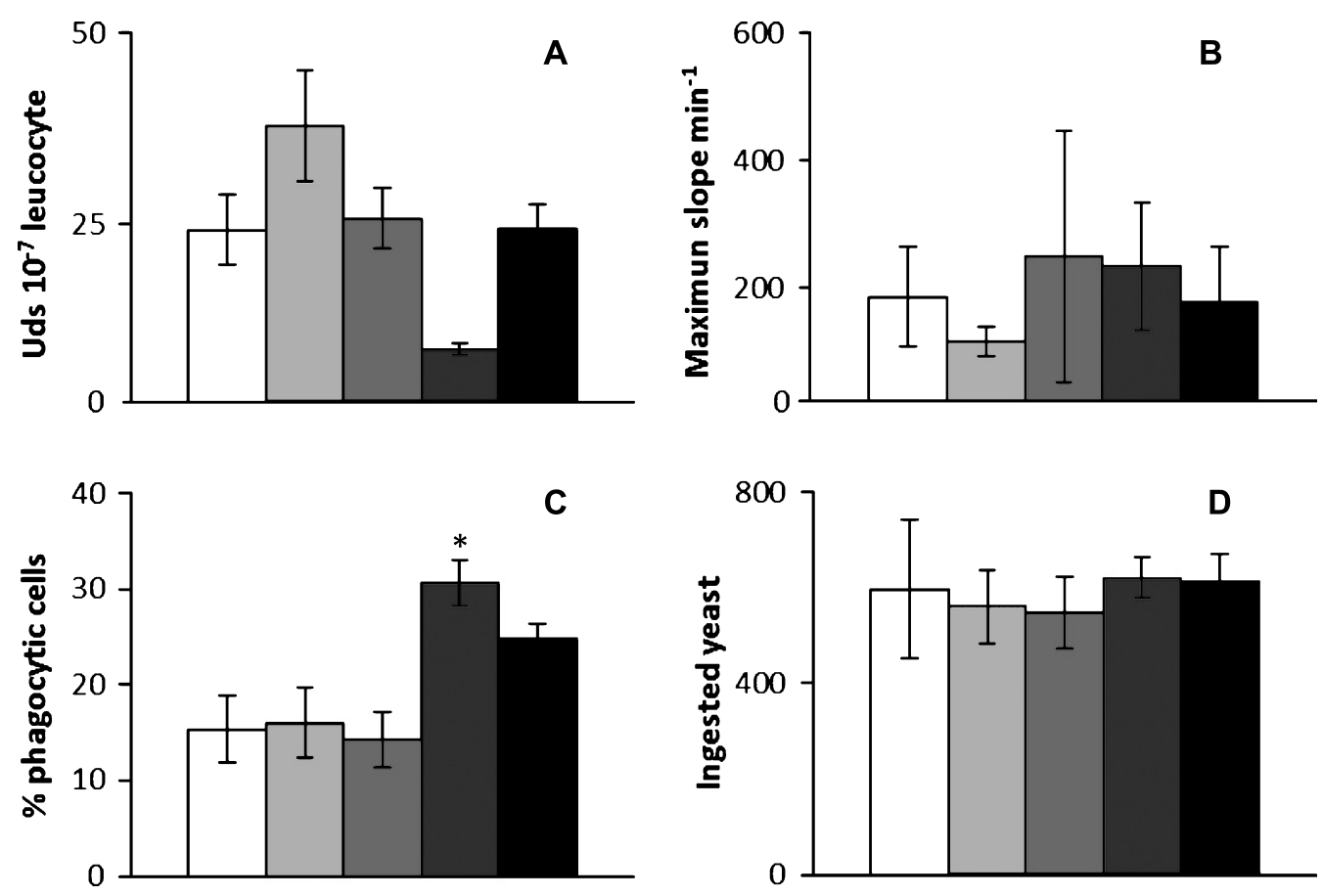

Fig. 3. Cellular innate immune activities of gilthead seabream head kidney leucocytes after being incubated with: ( $\square$ ) control,

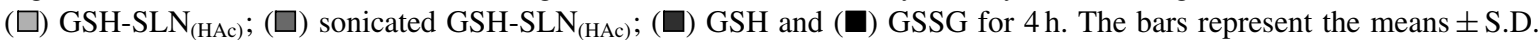
$(n=6)$. Asterisks denote significant differences between control and treated groups $(P \leq 0.05)$.

peroxidase content present on HK leucocytes nor in the respiratory burst activity of such cells, respect to the values obtained for control leucocytes (incubated only with culture cell medium) (Fig. 3A, B). However, the phagocytic ability of HK leucocytes previously incubated with GSH was significantly increased respect to the values found for control HK leucocytes (Fig. 3C). Similarly, the phagocytic ability of HK leucocytes incubated with GSSG was also increased, respect to the values found in control leucocytes, but the increments were not statistically significant. No significant deviations were observed in this parameter when HK leucocytes were incubated with either GSH-SLN HAc $_{(}$or sonicated $\mathrm{GSH}_{-\mathrm{SLN}_{\text {(HAc }}}$ (Fig. 3C). Curiously, the phagocytic capacity of gilthead seabream HK leucocytes was not affected by the incubation with sonicated and non-sonicated GSH-SLN $\mathrm{HAc}_{(\mathrm{H})}$ GSH or GSSG for $4 \mathrm{~h}$ (Fig. 3D). 


\section{Discussion}

Colloidal carriers based-formulations were already seen as promising platform for the delivery of biologically active compounds to cell fish [11]. In this context, the supply of antioxidant agents can significantly contribute to fish health, and by the use of colloidal carriers such active principles may be addressed to the immunocompetent HK leucocytes. Previously, to provide antioxidant agents plus other active substances for fish nutrition, their diet was improved thanks to the introduction of microalgae [26] or live feed components [27].

In the present work, we aimed at evaluating the performances of SLN based on a self-emulsifying lipid for the delivery of a well-known antioxidant peptide as GSH.

For this purpose, we selected the melt-emulsification method coupled with UltraTurrax homogenizer considering the self emulsifying properties of the lipid Gelucire ${ }^{\circledR} 50 / 13$ [13] which could help the entrapment of the hydrophilic GSH. Moreover, taking into account that the control of $\mathrm{pH}$ value of the aqueous phase is crucial for maintaining the active form of GSH instead of the inactive GSSG [28], herein we have focused on the effect of an aqueous phase of the starting emulsion constituted in one case by double distilled water [i.e., $\mathrm{GSH}_{-} \mathrm{SLN}_{(\mathrm{w})}$ ] and in the other case by diluted acetic acid $\left[\mathrm{GSH}_{-} \mathrm{SLN}_{(\mathrm{HAc})}\right]$. By keeping constant the amount of GSH initially loaded in the particles (i.e., $6 \mathrm{mg}$ ), it was observed that the highest A.E.\% (82.7\%) occurred using GSH-SLN (HAc) $_{\text {(Table 1). This }}$ finding can be related to the greater stability of GSH towards oxidation to disulfide GSSG in acidic medium. Therefore, the approach of sonication after particle production was carried out on GSH-SLN (HAc) rather than on GSH-SLN $(w)$. To identify the optimal conditions of sonication in terms of time and pulse frequency, two objectives were focused on: i.) the reduction of average particle size and ii.) the exclusion of any chemical modification of GSH, by monitoring its HPLC peak before and after each sonication treatment. In the literature, several examples of sonication applied to solid lipid nanoparticles are reported, either for loading of hydrophilic drug substances $[25,29]$ or hydrophobic ones [30-32]. Herein, we have tried to pre-form SLN according to the melt-emulsification technique and as a following step to apply probe-sonication, in an attempt to protect the sensitive molecule of GSH inside a preformed SLN carrier. On the other hand, by working according $1 \mathrm{~min}$ at $20 \mathrm{~W}$ of output of energy of the experimental set up, we prevented the risk of disruption of the outer core of lipid, which occurs in the presence of longer time of sonication/higher output of energy [33]. Upon our conditions of sonication, sonicated GSH-SLN $(\mathrm{HAc})$ did not exhibit any trace of metallic pollution from the probe and provided average diameter of $69 \mathrm{~nm}$ even though a partial decrease in A.E.\% was observed (i.e., $53 \%)$. Therefore, the sonicated formulation together with non-sonicated GSH-SLN $(\mathrm{HAc})$ were chosen as the two most promising formulations for following in vitro studies.

As well as for oral absorption in humans, also for fish absorption is it crucial to examine the amount of active principle delivered in the two main compartments of the digestive tract and, thus, both formulation containing GSH were exposed to two buffer media in order to mimic the fish stomach $(\mathrm{pH}=5)$ and the fish mucosa $(\mathrm{pH}=7)$ (Fig. 2A and B). Sonicated GSH-SLN (HAc) revealed sustained release profiles in both media without enzymes and the possible explanation can be that GSH is located mainly inside the lipid matrix as deduced from the Triton X assay. On the other hand, the release profiles suggested that the two types of SLN may protect GSH from the $\mathrm{pH}$ injuries of the milieu and, in this way, the vehicles may bypass biological barriers and, then, administer the antioxidant to gilthead seabream HK leucocytes.

As it has been previously mentioned, GSH is essential for cellular homeostasis and redox state and plays a vital role in diverse cellular functions. GSH also plays a role in the normal function of the immune system [34] because it regulates innate immunity at various levels and GSH also acts a signaling molecule [35]. However, GSH dimer, known as GSSG, is an inactive product. For these main reasons, the in vitro effects of GSH, GSSG or the SLN tested in the present work (sonicated and non-sonicated 
GSH-SLN $(\mathrm{HAc})$ ) were studied on gilthead seabream HK leucocytes. According to the properties known for GSH and GSSG on mammals, it was expected that incubation of HK leucocytes with GSH but not with GSSG had immuno-stimulant properties on such cells. It was interesting to note that any of the assayed compounds had significant effects neither in the peroxidase content of HK leucocytes nor in the respiratory burst activity of such cells, respect to the values obtained for control leucocytes (incubated only with cell culture medium). It is known that peroxidase is an important enzyme with microbicidal properties which utilizes one of the oxidative radicals $\left(\mathrm{H}_{2} \mathrm{O}_{2}\right)$ to produce hypochlorous acid [36]. This process is extremely important for killing foreign microorganisms [37]. Furthermore, phagocytic leukocytes, when appropriately stimulated, display a respiratory burst in which they consume oxygen and produce superoxide anions. It can be assumed that the non-statistically significant results in these parameter find in the present work are due to the fact that GSH scavenges peroxide species which can be harmful to the cells [35]. Nevertheless, these results can also be due to the used administration time (4 hours). Perhaps shorter GSH, GSSG or SLN incubation times could give rise to higher (and significant) leucocyte peroxidase content respect to control leucocytes, although new studies will be needed to corroborate this hypothesis.

Phagocytosis is in fact a specific form of endocytosis involving the vesicular interiorization of particles. Phagocytosis is essentially a defensive reaction against infection and invasion of the body by foreign substances and, in the immune system, phagocytosis is a major mechanism used to remove pathogens and/or cell debris. For these reasons, phagocytosis in vertebrates has been recognized as a critical component of the innate and adaptive immune responses to pathogens. Present results demonstrate that GSH but not GSSG modulates the function of fish immune cells, increasing their phagocytic properties. Since many decades it is known that GSH homeostasis becomes critical during physiological events such as phagocytosis for human leucocytes because GSH simultaneously induce the assembly of microtubules and the production of agents like $\mathrm{H}_{2} \mathrm{O}_{2}$ that can oxidize GSH [38]. It was also demonstrated that monocytes have a specific GSH transporter that is triggered by the release of $\mathrm{H}_{2} \mathrm{O}_{2}$ during the respiratory burst and that induces the uptake of GSH into the cell. Such a mechanism has the potential to protect the phagocyte against oxidant damage [39]. Further research is needed to demonstrate if such mechanisms are also present on fish leucocytes.

The fact that GSH increase phagocytosis by fish leucocyte is a very important result because GSH is a precursor for S-nitrosoglutathione (GSNO), and GSNO may represent one of the most important active forms of nitric oxide as an antimicrobial agent [40]. More concretely, Spallholz [41] observed that GSH "is structurally similar to the precursor of the antibiotics produced in fungi in the genera Penicillium and Cephalasporium". Future studies could evaluate the direct antimicrobial effects of GSH on bacterial fish pathogens that causes important diseases and, for this reason, important economic losses in fish aquaculture farms. It will be also very interesting to study the ability of GSH to enhance the activation of other immune cells, for example, fish lymphocytes, as occurs in mammals [42].

Finally, no statistically significant differences were observed on the phagocytic activity of HK leucocytes after being incubated with GSH-SLN $(\mathrm{HAc})$ or sonicated GSH-SLN $\mathrm{HAc}_{(\mathrm{H})}$. We know from a previous work that the ability of gilthead seabream HK leucocytes to internalize the SLN varied depending on both the incubation time and the SLN concentration. Furthermore, we demonstrated by using 6-Coumarin loaded SLN that after incubation with HK leucocytes, SLN appeared homogeneously distributed inside the leucocyte cytoplasm, as it was demonstrated by confocal microscopy because the green fluorescence of 6-Coumarin [10]. According to those results, in the present study we test the same SLN concentration and incubation time but 6-Coumarin was replaced with GSH, by achieve a site-specific targeting to fish immune systems. Nevertheless, no stimulant properties were observed after incubation of HK leucocytes with SLN (both sonicated and non-sonicated) which could be due to the fact that the highest values of HK leucocytes internalized 6-Coumarin SLN particles were only around 16\% [10]. Perhaps this small percentage of HK leucocytes (around 16\%) was stimulated 
although we quantified the total activity of the leucocyte population. Alternatively, to account for the results it can be hypothesized that the low amount of peptide released during the incubation time in which the cell viability is strictly maintained (i.e., 4 hours) resulted not sufficient to modify the in vitro phagocytic ability of HK leucocytes previously incubated with both the GSH-SLN examined.

\section{Conclusions}

We have formulated novel SLN based on the self-emulsifying lipid Gelucire ${ }^{\circledR}$ 50/13 intended as colloidal vector for the administration of little peptides as the antioxidant GSH to HK leucocytes immunocompetent fish cells. The mild preparative technique of melt-emulsification provided particles characterized by good A.E.\% in peptide and even below $100 \mathrm{~nm}$ in size as in the case of sonicated $\mathrm{GSH}_{-} \mathrm{SLN}_{(\mathrm{HAc})}$. Moreover, these nanocarriers maintained their features of size and surface charge up to three months after storage at $4^{\circ} \mathrm{C}$. However, the peptide GSH localization seems to be inside the Gelucire $^{\circledR}$ 50/13 lipid wall. From the biological evaluation of SLN, it was deduced that the phagocytic ability (the main innate cellular immune response) of HK leucocytes previously incubated with GSH was significantly increased respect to the values found for control. In conclusion, this article provides detailed information on the immuno-stimulant effects of GSH on fish leucocytes. This result along with its therapeutic and protective effects makes GSH a key molecule to the fish immune system and health.

The present work constitutes an initial approach to consider SLN as nanocarriers for delivering biologically active substances to fish immune cells. Further studies are required to elucidate the advantage of using this formulation in aquaculture.

\section{Conflict of interest}

The authors have no financial, consulting or personal relationships with other people or organizations which could have influenced the present work.

\section{Acknowledgments}

A.T., D.M and G.T. would like to acknowledge Dr. Claudio Perego (Gattefossè, Italy) for his important help with to provide lipids. PONa300369 "Laboratorio per lo Sviluppo Integrato delle Scienze e delle Tecnologie dei Materiali Avanzati e per dispositivi innovativi-LABORATORIO SISTEMA” was also aknowledged. Financial support by grant AGL2014-51839-C5-1-R (Spanish Ministry of Economy and Competitiveness and ERDF) and 19883/GERM/15 (Grupo de Excelencia, de la Fundación Séneca de la Región de Murcia, Spain) is gratefully acknowledged by M.A.E.

\section{References}

[1] Reen FJ, Gutiérrez-Barranquero JA, Dobson ADW, Adams C, O’Gara F. Emerging concepts promising new horizons for marine biodiscovery and synthetic biology. Mar Drugs 2015;13:2924-54.

[2] Espín S, Martínez-López E, Jiménez P, María-Mojica P, García-Fernández AJ. Interspecific differences in the antioxidant capacity of two Laridae species exposed to metals. Environ Res 2016;147:115-24.

[3] Lemaire B, Mignolet E, Debier C, Calderon PB, Thomé JP, Rees JF. High hydrostatic pressure influences the in vitro response to xenobiotics in Dicentrarchus labrax liver. Aquat Toxicol 2016;173:43-52.

[4] Javed M, Ahmad I, Usmani N, Ahmad M. Bioaccumulation, oxidative stress and genotoxicity in fish (Channa punctatus) exposed to a thermal power plant effluent. Ecotoxicol Environ Saf 2016; 127:163-9. 
[5] Paolucci M, Fasulo G, Volpe MG. Employment of marine polysaccharides to manufacture functional biocomposites for aquaculture feeding applications. Mar Drugs 2015;13:2680-93.

[6] Zhang N, Ping Q, Huang G, Xu W, Cheng Y, Han X. Lectin-modified solid lipid nanoparticles as carriers for oral administration of insulin. Int J Pharm 2006;327:153-9.

[7] Kumar S, Randhawa JK. High melting lipid based approach for drug delivery: Solid lipid nanoparticles. Mater Sci Eng C 2013;33:1842-52.

[8] Almeida AJ, Souto E. Solid lipid nanoparticles as a drug delivery system for peptides and proteins. Adv Drug Deliv Rev 2007;59:478-90.

[9] Matougui N, Boge L, Groo AC, Umerska A, Ringstad L, Bysell H, Saulnier P. Lipid-based nanoformulations for peptide delivery. Int J Pharm 2016;17502:80-97. doi: 10.1016/j.ijpharm.2016.02.019

[10] Trapani A, Mandracchia D, Di Franco C, Cordero H, Morcillo P, Comparelli R, Cuesta A, Esteban MA. In vitro characterization of 6-Coumarin loaded solid lipid nanoparticles and their uptake by immunocompetent fish cells. Colloids and Surfaces: Biointerfaces 2015;127:79-88.

[11] Shaalan M, Saleh M, El-Mahdy M, El-Matbouli M. Recent progress in applications of nanoparticles in fish medicine: A review. Nanomedicine: NBM 2015;xx:1-10, http://dx.doi.org/10.1016/j.nano.2015.11.005

[12] Date AA, Vador N, Jagtap A, Nagarsenker MS. Lipid nanocarriers (GeluPearl) containing amphiphilic lipid Gelucire 50/13 as a novel stabilizer: Fabrication, characterization and evaluation for oral drug delivery. Nanotechnology 2011;22:275102.

[13] Jeon HS, Seo JE, Kim MS, Kang MH, Oh DH, Jeon SO, Seong HJ, Choi YW, Lee S. A retinyl palmitate-loaded solid lipid nanoparticle system: Effect of surface modification with dicetyl phosphate on skin permeation in vitro and anti-wrinkle effect in vivo. Int J Pharm 2013;452:311-20.

[14] Ibrahim WM, AlOmrani AH, Yassin AE. Novel sulpiride-loaded solid lipid nanoparticles with enhanced intestinal permeability. Int J Nanomedicine 2014;9:129-44.

[15] Woo JO, Misran M, Lee PF, Tan LP. Scientific World Journal 2014;205703.

[16] Trapani A, Di Gioia S, Ditaranto N, Cioffi N, Goycoolea FM, Carbone A, Garcia-Fuentes M, Conese M, Alonso MJ. Systemic heparin delivery by the pulmonary route using chitosan and glycol chitosan nanoparticles. Int $\mathrm{J}$ Pharm 2013;447:115-23.

[17] Su L, Zhaoshuai J, Zou MJ, Nie X, Shi Y, Cheng G. Preparation, characterization, pharmacokinetics and tissue distribution of solid lipid nanoparticles loaded with tetrandrine. AAPS Pharm Sci Tech 2011;12:1011-8.

[18] Montenegro L, Trapani A, Fini P, Mandracchia D, Latrofa A, Cioffi N, Chiarantini L, Piccieri GG' Brundu S' Puglisi G. Chitosan nanoparticles for topical co-administration of the antioxidants glutathione and idebenone: Characterization and in vitro release. British Journal of Pharmaceutical Technology 2014;4:2387-2406.

[19] Fir MM, Milivojevic L, Prosek M, Smidovnik A. Properties Studies of Coenzyme Q10-Cyclodextrins complexes. Acta Chim Slov 2009;56:885-91.

[20] Das D, Nath BC, Phukon P, Kalita A, Dolui SK. Synthesis of ZnO nanoparticles and evaluation of antioxidant and cytotoxic activity. Colloids Surf. B Biointerfaces 2013;111:556-60.

[21] Esteban MA, Mulero V, Muñoz J, Meseguer J. Methodological aspects of assessing phagocytosis of Vibrio anguillarum by leucocytes of gilthead seabream (Sparus aurata L.) by flow cytometry and electron microscopy. Cell Tissue Res 1998;293:133-41.

[22] Quade MJ, Roth JA. A rapid, direct assay to measure degranulation of bovine neutrophil primary granules. Vet Immunol Immunopathol 1997;58:239-48.

[23] Bayne CJ, Levy S. Modulation of the oxidative burst in trout myeloid cells by adrenocorticotropic hormone and catecholamines: Mechanisms of action. J Leukoc Biol 1991;50:554-60.

[24] Rodríguez A, Esteban MA, Meseguer J. Phagocytosis and peroxidase release by seabream (Sparus aurata L.) leucocytes in response to yeast cells. Anat Rec A Discov Mol Cell Evol Biol 2003;272:415-23.

[25] Shah B, Khunt D, Bhatt H, Misra M, Padh H. Application of quality by design approach for intranasal delivery of rivastigmine loaded solid lipid nanoparticles: Effect on formulation and characterization parameters. Eur J Pharm Sci 2015;78:54-66.

[26] Cerezuela R, Guardiola FA, Meseguer J, Esteban MA. Enrichment of gilthead seabream (Sparus aurata L.) diet with microalgae: Effects on the immune system. Fish Physiol Biochem 2012;38:1729-39.

[27] Fletcher Jr RC, Roy W, Davie A, Taylor J, Robertson D, Migaud H. Evaluation of new microparticulate diets for early weaning of Atlantic cod (Gadus morhua): Implications on larval performances and tank hygiene. Aquculture 2007;263:35-51.

[28] Camera E, Picardo M. Analytical methods to investigate glutathione and related compounds in biological and pathological processes. J Chromatogr B 2002;781:181-206.

[29] Becker Peres L, Becker Peres L, de Araújo PH, Sayer C. Solid lipid nanoparticles for encapsulation of hydrophilic drugs by an organic solvent free double emulsion technique. Colloids Surf. B Biointerfaces 2016;140:317-23. 
[30] Kurakula M, Ahmed OA, Fahmy UA, Ahmed TA. Solid lipid nanoparticles for transdermal delivery of avanafil: Optimization, formulation, in-vitro and ex-vivo studies. J Liposome Res 2016;19:1-9.

[31] Esmaeili M, Aghajani M, Abbasalipourkabir R, Amani A. Budesonide-loaded solid lipid nanoparticles for pulmonary delivery: Preparation, optimization, and aerodynamic behavior. Artif Cells Nanomed Biotechnol 2016;13:1-8.

[32] Chaudhary S, Garg T, Murthy RS, Rath G, Goyal AK. Development, optimization and evaluation of long chain nanolipid carrier for hepatic delivery of silymarin through lymphatic transport pathway. Int J Pharm 2015;485:108-21.

[33] Taurozzi JS, Hackley VA, Wiesner MR. Ultrasonic dispersion of nanoparticles for environmental, health and safety assessment-issues and recommendations. Nanotoxicology 2011;5:711-29.

[34] Buhl R, Holroyd K J, Mastrangeli A, Cantin AM, Jaffe HA, Wells FB, Saltini C, Crystal RG. Systemic glutathione deficiency in symptom-free HIV-seropositive individuals. Lancet 1989;II:1294-98.

[35] Ghezzi P. Role of glutathione in immunity and inflammation in the lung. Int J Gen Med 2011;4:105-13.

[36] Dalmo R, Ingebrigtsen K, Bøgwald J. Non-specific defence mechanisms in fish, with particular reference to the reticuloendothelial system (RES). J Fish Dis 1997;20:241-73.

[37] Johnston R Jr. Oxygen metabolism and the microbicidal activity of macrophages. Federation Proceedings 1978;2759-64.

[38] Oliver JM, Albertini DF, Berlin RD. Effects of glutathione-oxidizing agents on microtubule assembly and microtubuledependent surface properties of human neutrophils. J Cell Biol 1976;71:921-32.

[39] Seres T, Knickelbein RG, Warshaw JB,Johnston RB Jr. The phagocytosis-associated respiratory burst in human monocytes is associated with increased uptake of glutathione. J Immunol 2000;165:3333-40.

[40] MacMicking J, Xie QW, Nathan C. Nitric oxide and macrophage function. Ann Rev Immunol 1997;15:323-50.

[41] Spallholz JE. Glutathione: Is it an evolutionary vestige of the penicillins? Med Hypoth 1987;23:253-57.

[42] Guerra C, Morris D, Sipin A, Kung S, Franklin M, Gray D, Tanzil M, Guilford F, Khasawneh FT, Venketaraman V. Glutathione and adaptive immune responses against Mycobacterium tuberculosis infection in healthy and HIV infected individuals. PLoS One 2011;6: e28378. doi: 10.1371/journal.pone.0028378 